\title{
A Simplified Generic Optimum Power Control scheme for CDMA Cellular Systems
}

\author{
Jad Nasreddine \\ Multimedia Networks and Services Department \\ GET / ENST de Bretagne \\ 2 rue de la châtaigneraie, CS 17607 \\ 35576 Cesson Sévigné Cedex, France \\ e-mail: jad.nasreddine $($ enst-bretagne.fr
}

\author{
Xavier Lagrange \\ Multimedia Networks and Services Department \\ GET / ENST de Bretagne \\ 2 rue de la châtaigneraie, CS 17607 \\ 35576 Cesson Sévigné Cedex, France \\ e-mail: xavier.lagrange $@$ enst-bretagne.fr
}

\begin{abstract}
Power control is of great importance in reducing cochannel interference and increasing the capacity of mobile telecommunications systems. The aim of optimum power control procedure is to achieve a maximum Carrier-to-Interference Ratios $(C I R)$ in all active communication links (i.e. $C I R$ balancing). Optimum power control with $C I R$ balancing has been widely studied for Frequency-Division/Time-division Multiple Access (FDMA/TDMA) cellular systems. In these systems, the $C I R$ balanced optimum power control was transformed to an eigenvalue problem using link-gain matrix. The same method was proposed to CDMA systems. However, the size of the linkgain matrix is proportional to the square of the number of communication links in the system. Thus, the computation of the eigenvalue may be infeasible in CDMA systems with high load. Moreover, optimum power control has never been studied in crossed slots where some mobiles are active in downlink and other in uplink. A simplified optimum power control scheme has been proposed to reduce the link-gain matrix only in the forward link. In this paper, we introduce a simplified generic optimum power control scheme for uplink, downlink and crossed slots. This scheme is based on a link-gain matrix whose size is proportional to the square of the number of cells. Simulation results have justified that the proposed scheme achieves the same upper bound of $C I R$ as the complex scheme.
\end{abstract}

\section{INTRODUCTION}

The CDMA technique will be used in the air interface of the third generation mobile telecommunications systems to increase system capacity. The capacity of CDMA systems is limited by intercell and intracell interference [1]; therefore, power control is essential in the design of these systems. The measure of quality in cellular system design is the $C I R$ ratio [2]. Aein has introduced the concept of $C I R$ balancing to investigate cochannel interference management in satellite systems [3]. In this concept, all active communication links must be served with the same $C I R$. As in satellite systems, the $C I R$-balanced power control for FDMA/TDMA systems has been formulated as an eigenvalue problem in [2].

In each cell of a CDMA system, the radio bandwidth is shared by several mobiles, which results in a three dimensional link-gain matrix. However, $C I R$ balancing is not an eigenvalue problem unless the link-gain matrix has only two dimensions [4]. The three dimensional matrix has been simplified to a two dimensional matrix when intracell $C I R$ balancing was used

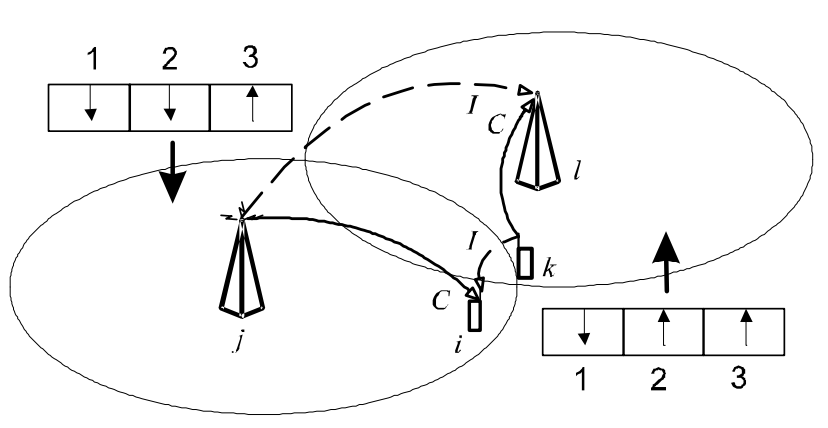

Fig. 1. Crossed time slot (2) in a sytem with two cells.

[4]. Moreover, a complex link-gain matrix was introduced in [5] for crossed slots, where some mobiles are active in uplink and other are active in downlink.

The maximum achievable $C I R$ of a cochannel is deduced from the maximum positive eigenvalue of the link-gain matrix. The dimension of the link-gain matrices used in [4] and [5] are proportional to the square of the number of active communication links. The eigenvalue computation of these matrices may be infeasible due to the exceedingly matrix large size. To reduce the matrix dimension in the forward link, the optimal power control is applied to the total transmitted power by base stations and not to the power of each communication link [6][7][8][9].

In Europe and in several other countries, Universal Mobile Telecommunications System (UMTS) will be used. The UMTS air interface includes both Frequency Division Duplex (FDD) and Time Division Duplex (TDD) modes. The transmission unit in the UMTS air interface is a $10-\mathrm{ms}$ frame. Each frame is divided into 15 time slots. To increase the flexibility of the TDD mode, some slots may be used for uplink in some cells and for downlink in other cells (fig. 1). In these slots, denoted crossed slots, mobile-to-mobile and base station-to-base station interferences appear. The performance of crossed slots under optimal power control has not been investigated, as far as we know. In this paper, we study the performance of a simplified 
generic optimal power control during uplink, downlink and crossed slots in the TDD mode of UMTS. The same optimal power control can be used in the FDD mode.

In the next section, the system model is described. The optimal power control is presented and justified in section 3 . Simulation results are drawn in section 4 . The last section presents some concluding remarks and future works.

\section{SySTEM MODEL}

The optimal power control is evaluated in a simulation platform consisting of square-shaped cells [4]. A wraparound technique is applied to ensure that each cell is completely surrounded by a symmetric pattern. All link gains are assumed to be known through intelligent measuring. The number of mobiles in a cell $j$ during a time slot $n$ is denoted $N_{j, n}$. We suppose that a mobile is always served by the best-received base station and that joint detection technique is used [10]. We denote $\beta_{d}$ and $\beta_{u}$ the factors of remaining intracell interferences respectively in downlink and in uplink after the joint detection procedure. Thus, the interference power estimated at the receiver after the joint detection is given by:

$$
I=\beta_{\ell} \cdot I_{\text {intra }}+I_{\text {inter }}
$$

where $\ell$ is the link direction type (i.e. $\ell=d$ for downlink and $\ell=u$ for uplink), $I_{\text {intra }}$ and $I_{\text {inter }}$ are the intracell and intercell interference powers.

We assume that the interference power is much larger than the white noise in the receiver; therefore, the white noise is not considered in our study. We also assume that all mobiles request the same service.

If a base station $j$ is transmitting during a time slot $n$, the corresponding cell is called downlink-cell. If the mobiles of a cell $j$ are transmitting during a time slot $n$, the cell $j$ is called uplink-cell. In crossed slots, uplink and downlinkcells are randomly distributed with an asymmetry rate $a_{r}=$ $C_{u} /\left(C_{d}+C_{u}\right)$, where $C_{u}$ and $C_{d}$ are the number of uplink and downlink cells respectively.

Indexes $i$ and $k$ always refer to mobiles. Index $j$ refers to the base station that serves the studied mobile while index $l$ refers to a neighboring cell (fig. 1). The set of base station indexes is denoted $\Pi=\{1, \cdots, M\}$ and the set of mobiles connected to the base station $j$ during slot $n$ is denoted $S_{j, n}$.

In a downlink-cell $j$, the useful power $C_{\mathrm{M}, i, n}$ received by mobile $i$ during the time slot $n$ is:

$$
C_{\mathrm{M}, i, n}=G_{i, j} P_{\mathrm{B}, i, n}
$$

where $P_{\mathrm{B}, i, n}$ is the power transmitted by base station $j$ to mobile $i$ during time slot $n$ and $G_{i, j}$ is the path gain between mobile $i$ and its server $j$.

The normalized path gain $Z_{i, l}$ between mobile $i$ and a neighboring base station $l$ is defined by:

$$
Z_{i, l}=\frac{G_{i, l}}{G_{i, j}} .
$$

A base station $j$ transmits a total power $P_{\mathrm{T}, j, n}$ during time slot $n$. This power is distributed between the mobiles of cell $j$ such that every mobile $i$ receives:

$$
C_{\mathrm{M}, i, n}=G_{i, j} \alpha_{i, n} P_{\mathrm{T}, j, n}
$$

where $\alpha_{i, n}$ is the portion of $P_{\mathrm{T}, j, n}$ dedicated to mobile $i$ during time slot $n$. Thus:

$$
\sum_{i \in S_{j, n}} \alpha_{i, n}=1 \quad \forall j \in \Pi
$$

If the $C I R$-balanced power control is applied to cell $j$, all communication links must be received with the same $C I R$ [2][3]. Moreover, all communication links are interfered by the same intercell-interference in a given cell and during a given slot. Therefore, all mobiles of an uplink-cell $j$ must be received with the same power $C_{\mathrm{B}, j, n}$ by their server. The received power is:

$$
G_{i, j} P_{\mathrm{M}, i, n}=C_{\mathrm{B}, j, n} \quad \forall i \in S_{j, n}
$$

where $P_{\mathrm{M}, i, n}$ is the power transmitted by mobile $i$. The value of $C_{\mathrm{B}, j, n}$ is not the same in all cells and is fixed by the power control scheme.

\section{OPTIMAL POWER CONTROL IN A TIME SLOT}

We suppose that time slot allocation is done and we analyze the optimal power control in a crossed time slot. Uplink and downlink time slots are special cases of crossed time slots and thus, only crossed slots are investigated in this paper. We first estimate the $C I R$ in downlink- and uplink-cells independentely as a function of the total intereference profile. The $C I R$-balanced power control problem is then formulated as a eigenvalue problem by combining the linear eaquations of downlink- and uplink-cells.

In a crossed time slot $n$, let $\Pi_{d, n}$ and $\Pi_{u, n}$ be the sets of downlink-cells and uplink-cells respectively. Our objective is to find the highest $C I R$ that can be achieved by all communication links in the system. This $C I R$ is reached when the optimum power control is used during each time slot independently. Therefore, only one time slot is studied.

In downlink-cells, the power control scheme is applied to the total power transmitted by base stations instead of specific power to each mobile. In uplink-cells, the power control scheme is applied to the received power in base stations. In crossed time slots, each type of link is studied independently. Equations obtained in both links are combined to form one linear system.

Let $\gamma_{n}$ be the $C I R$ that can be reached by all active mobiles during a time slot $n$ :

$$
\Gamma_{i, j, n} \geq \gamma_{n} \quad \forall i \in S_{j, n}, j \in \Pi
$$

where $\Gamma_{i, j, n}$ is the estimated $C I R$ of mobile $i$. 


\section{A. Downlink-cells}

The path gain between two mobiles $i$ and $k$ is denoted $G_{k, i}$. From equation (6), the power transmitted by mobile $k$ of cell $l$ is $C_{\mathrm{B}, l, n} / G_{k, l}$. Thus, the $C I R$ of a mobile $i$ in a downlink-cell $j$ is given by:

$$
\Gamma_{i, j, n}=\frac{\alpha_{i, n} G_{i, j} P_{\mathrm{T}, j, n}}{I_{\mathrm{d}, i, n}}
$$

where $I_{\mathrm{d}, i, n}$ is the total interference power received by mobile $i$ during time slot $n$ :

$$
\begin{aligned}
I_{\mathrm{d}, i, n}= & \underbrace{\beta_{d} G_{i, j}\left(1-\alpha_{i, n}\right) P_{\mathrm{T}, j, n}}_{\text {IntraI }}+\underbrace{\sum_{l \in \Pi_{d, n}-\{j\}} G_{i, l} P_{\mathrm{T}, l, n}}_{\text {InterID }} \\
& +\underbrace{\sum_{l \in \Pi_{u, n}}\left(\sum_{k \in S_{l, n}} \frac{G_{k, i}}{G_{k, l}}\right) C_{\mathrm{B}, l, n}}_{\text {InterIU }}
\end{aligned}
$$

where IntraI is the intracell-interference power, InterID is the intercell-interference power received from neighboring base stations active in downlink and InterIU is the intercellinterference power received from all neighboring mobiles active in the uplink.

By substituting the value of $\Gamma_{i, j, n}$ from (8) in (7) and using (3), we obtain:

$$
\begin{aligned}
\frac{\alpha_{i, n}}{\gamma_{n}} P_{\mathrm{T}, j, n} \geq & \beta_{d}\left(1-\alpha_{i, n}\right) P_{\mathrm{T}, j, n}+\sum_{l \in \Pi_{d, n}-\{j\}} Z_{i, l} P_{\mathrm{T}, l, n} \\
& +\sum_{l \in \Pi_{u, n}}\left(\sum_{k \in S_{l, n}} \frac{G_{k, i}}{G_{i, j} G_{k, l}}\right) C_{\mathrm{B}, l, n}
\end{aligned}
$$

By making the sum over all mobiles of the cell $j$ and adding $P_{\mathrm{T}, j, n}$ to both sides of the last inequality, we obtain:

$$
\frac{1+\gamma_{n}}{\gamma_{n}} P_{\mathrm{T}, j, n} \geq P_{\mathrm{T}, j, n}+\Im_{\mathrm{d}, j, n}
$$

where $\Im_{\mathrm{d}, j, n}$ is given by:

$$
\begin{aligned}
\Im_{\mathrm{d}, j, n}= & \beta_{d}\left(N_{j, n}-1\right) P_{\mathrm{T}, j, n} \\
& +\sum_{l \in \Pi_{d, n}-\{j\}}\left(\sum_{i \in S_{j, n}} Z_{i, l} P_{\mathrm{T}, l, n}\right) \\
& +\sum_{l \in \Pi_{u, n}}\left(\sum_{i \in S_{j, n}} \sum_{k \in S_{l, n}} \frac{G_{k, i}}{G_{i, l} G_{k, l}}\right) C_{\mathrm{B}, l, n} .
\end{aligned}
$$

If the intracell and intercell $C I R$-balanced power control is used, the set of relations (11) in all cells gives the same achievable $C I R$ as in [4] which is the maximum achievable $C I R$, because there is only one optimum solution for (7) [2].

\section{B. Uplink-cells}

The $C I R$ of a mobile $i$ in an uplink-cell $j$ is:

$$
\Gamma_{i, j, n}=\frac{C_{\mathrm{B}, j, n}}{I_{\mathrm{u}, j, n}},
$$

where $I_{\mathrm{u}, j, n}$ is the total interference power corresponding to mobile $i$ during time slot $n$. This interference power is the same for all mobiles of cell $j$ :

$$
\begin{aligned}
I_{\mathrm{u}, j, n}= & \underbrace{\beta_{u}\left(N_{j, n}-1\right) C_{\mathrm{B}, j, n}}_{\text {IntraI }}+\underbrace{\sum_{l \in \Pi_{d, n}} G_{j, l} P_{\mathrm{T}, l, n}}_{\text {InterID }} \\
& +\underbrace{\sum_{l \in \Pi_{u, n}-\{j\}}\left(\sum_{k \in S_{l, n}} Z_{k, j} C_{\mathrm{B}, l, n}\right)}_{\text {InterIU }} .
\end{aligned}
$$

By substituting the value of $\Gamma_{i, j, n}$ from (13) in (7) and adding $C_{\mathrm{B}, j, n}$ to both sides of the last inequality we obtain:

$$
\frac{1+\gamma_{n}}{\gamma_{n}} C_{\mathrm{B}, j, n} \geq C_{\mathrm{B}, j, n}+I_{\mathrm{u}, j, n}
$$

\section{Generic model}

By combining equations (11) for all downlink-cells and (15) for all uplink-cells, we obtain a linear system which can be represented by the following matrix inequality:

$$
\mathbf{Z}^{(n)} \mathbf{R}_{n} \leq \frac{1+\gamma_{n}}{\gamma_{n}} \mathbf{R}_{n}
$$

where $\mathbf{R}_{n}=\left[R_{j, n}\right]$ is the $M \times 1$ vector with elements:

$$
R_{j, n}= \begin{cases}P_{\mathrm{T}, j, n} & \forall j \in \Pi_{d, n} \\ C_{\mathrm{B}, j, n} & \forall j \in \Pi_{u, n}\end{cases}
$$

$\mathbf{Z}^{(n)}$ is an $M \times M$ non negative matrix with elements:

$$
Z_{j, l}^{(n)}= \begin{cases}\sum_{i \in S_{j, n}} Z_{i, l} & \forall j, l \in \Pi_{d, n}, j \neq l \\ 1+\beta_{d}\left(N_{j, n}-1\right) & \forall j, l \in \Pi_{d, n}, j=l \\ \sum_{i \in S_{j, n}} \sum_{k \in S_{l, n}} \frac{G_{k, i}}{G_{i, j} G_{k, l}} & \forall j \in \Pi_{d, n}, l \in \Pi_{u, n} \\ G_{j, l} & \forall j \in \Pi_{u, n}, l \in \Pi_{d, n} \\ 1+\beta_{u}\left(N_{j, n}-1\right) & \forall j, l \in \Pi_{u, n}, j=l \\ \sum_{k \in S_{l, n}} Z_{k, j} & \forall j, l \in \Pi_{u, n}, j \neq l\end{cases}
$$

It must be noted that vector $R_{n}$ includes the total base station transmitted powers in downlink-cells and the base station received powers in uplink-cells. Moreover, the simplified link-gain matrix is always an $M \times M$ matrix, whatever the number of mobiles in the different cells. On the contrary, the size of the normal link-gain matrix used in [4] and [5] is $\sum_{j \in \Pi} N_{j, n} \times \sum_{j \in \Pi} N_{j, n}$ during a time slot $n$. Thus, the size of the latter matrix may be very large compared to the proposed link-gain matrix dimensions. The reduced size of the simplified matrix leads to an important complexity decrease when compared to the case with the normal matrix, especially in systems with high loads.

From the Perron Frobenius theorem [11], the maximum value $\gamma_{n}^{*}$ of $\gamma_{n}$ that can be reached during a time slot $n$ with positive powers is obtained when the matrix inequality (16) became an equality:

$$
\mathbf{Z}^{(n)} \mathbf{R}_{n}^{*}=\frac{1+\gamma_{n}^{*}}{\gamma_{n}^{*}} \mathbf{R}_{n}^{*}
$$


and the value of $\gamma_{n}^{*}$ is given by the following equation:

$$
\gamma_{n}^{*}=\frac{1}{\lambda_{n}^{*}-1}
$$

where $\lambda_{n}^{*}$ is the largest real eigenvalue of the matrix $\mathbf{Z}^{(n)}$ and $\mathbf{R}_{n}^{*}$ is the corresponding eigenvector.

If the power vector $\mathbf{R}_{n}^{*}$ is used, all mobiles will have a $C I R$ equal to $\gamma_{n}^{*}$. Moreover, the transmitted power of a mobile in an uplink-cell $j$ is deduced from $C_{\mathrm{B}, j, n}$ using (6).

The following system is deduced from relations (8) and (19) in a cell $j$ :

$$
\gamma_{n}^{*}=\left\{\begin{array}{c}
\frac{\alpha_{i, n} P_{\mathrm{T}, j, n}}{I_{\mathrm{d}, i, n} / G_{i, j}} \\
\frac{P_{\mathrm{T}, j, n}}{\Im_{\mathrm{d}, j, n}}
\end{array},\right.
$$

From this system, we can deduce the value of $\alpha_{i, n}$, which is given by equation (22).

From equation (22), we can deduce that $\alpha_{i, n}$ is the ratio of the total power received by mobile $i$ to the sum of the total powers received by all mobiles of cell $j$.

If we suppose that we have the same mobile distribution and radio characteristics for uplink and downlink, the linkgain matrix elements during uplink and downlink slots are respectively given by:

$$
\begin{aligned}
& Z_{j, l}^{(u)}= \begin{cases}\sum_{k \in S_{l, n}} Z_{k, j} & \forall j, l \in \Pi_{u, n}, j \neq l \\
1+\beta\left(N_{j, n}-1\right) & \forall j, l \in \Pi_{u, n}, j=l\end{cases} \\
& Z_{j, l}^{(d)}= \begin{cases}\sum_{i \in S_{j, n}} Z_{i, l} & \forall j, l \in \Pi_{d, n}, j \neq l \\
1+\beta\left(N_{j, n}-1\right) & \forall j, l \in \Pi_{d, n}, j=l\end{cases}
\end{aligned}
$$

Therefore $Z_{j, l}^{(u)}=\sum_{k \in S_{l, n}} Z_{k, j}=Z_{l, j}^{(d)}$ when $j \neq l$ and $Z_{j, j}^{(u)}=Z_{j, j}^{(d)}$. Thus, the uplink link-gain matrix is the transpose of the downlink link-gain matrix. In this case, the maximum achievable $C I R$ in uplink and in downlink are identical and are deduced from the eigenvalue of (23) and (24). This result corresponds to the result presented by Zander for FDMA/TDMA systems [12].

\section{Simulations And Results}

To investigate the performance of the optimal power control procedure, a CDMA cellular system consisting of $5 \times 5$ blocks is considered. The block size is $200 \times 200$ meters. Only one slot of the TDMA frame is studied. It is supposed that all cells have the same number of simultaneous active mobiles and this number is limited to 16 as in the UMTS TDD mode. The values of $\beta_{d}$ and $\beta_{u}$ are respectively 0.1 and 0.2 . The orthogonality factor is higher in downlink, due to the synchronization between the different communication links in the downlink-cell.

The assumed propagation model is an Okumura-hata-cost 231 model with shadowing:

$$
P_{r}=P_{e} \frac{k}{d_{x, y}^{\gamma}} a_{x, y},
$$

where $P_{r}$ and $P_{e}$ are respectively the received and the transmitted powers, $k$ and $\gamma$ are propagation constants [13],
TABLE I

PROPAGATION MODEL CONSTANTS (FROM [13])

\begin{tabular}{|c|c|c|c|}
\cline { 2 - 4 } \multicolumn{1}{c|}{} & $B S-B S$ & $B S-M S$ & $M S-M S$ \\
\hline$k[\mathrm{~dB}]$ & -112.7 & -127.7 & -147.4 \\
\hline$\gamma[\mathrm{dB}]$ & 35.5 & 35.5 & 40 \\
\hline $\operatorname{var}\left[a_{x, y}\right][\mathrm{dB}]$ & 4.24 & 6 & 7.34 \\
\hline
\end{tabular}

which depend on the type of environment, and $d$ is the distance between the transmitter and the receiver. Factor $a_{x, y}$ models the shadowing effect. It is a time constant, Log-Normal variable with zero mean [14] (table I). The shadowing factor variance and the pathloss constant between two mobiles are very high compared to those between two base stations due to the low altitude of mobile antennas.

The normal link-gain matrices of uplink and downlink slots are the matrices used in [4], while the matrix in [5] is used as the normal link-gain matrix in crossed slots.

In table II the maximum achievable $C I R$ given by the simplified and the normal link-gain matrices for crossed slots with an asymmetry rate of 0.5 are presented. We can note that both methods give exactly the same maximum achievable $C I R$. Moreover, the same maximum achievable $C I R$ was obtained in all mobile distribution samples. However, the size of the simplified matrix is $25 \times 25$ while the size of the normal link matrix is an increasing function of the number of mobiles in cells. Therefore, the computation time of $\gamma^{*}$ with the simplified matrix is negligible counter the computation time with the normal matrix when the maximum load is used (i.e. the size of simplified and the normal matrix are respectively $25 \times 25$ and $400 \times 400$ for a load of 16 mobiles/cell/slot).

In figure 2, the average maximum achievable $C I R$ given by the simplified link-gain matrix is plotted for downlink, uplink and crossed slots. In crossed slots, the asymmetry rates 0.5 and 0.2 were used. The $95 \%$ confidence interval is also plotted in this figure. As expected, the uplink and the downlink slots have almost the same maximum $C I R$ while the crossed slot has a lower maximum $C I R$. The small difference between the results of uplink and downlink slots is due to the different impact of the intracell interference in the two link directions. Moreover, the degradation in the quality of service in crossed slots is due to high interference between close active mobiles in different link directions. This degradation is at its maximum when the asymmetry rate is 0.5 . The high variation in the maximum achievable $C I R$ in crossed slots is due to the high number of parameters affecting the path gain matrix. In uplink or downlink slots, only path gains between mobiles and base stations has an impact on the path gain matrix. In crossed slots however, new types of path gain (i.e. path gains between mobiles and path gains between base stations) appear. The path gains between mobiles are highly dependent on the mobile positions. Hence, the path gain matrix in crossed slots may change drastically dependent on the mobile positions. As a result of the large range of the upper bound $C I R$, we have noticed that in $14 \%$ of cases the maximum achievable $C I R$ in crossed slots was higher than in uplink slots when the asymmetry value 0.2 and 


$$
\alpha_{i, n}=\frac{\beta_{d} P_{\mathrm{T}, j, n}+\sum_{l \in \Pi_{d, n}-\{j\}} Z_{i, l} P_{\mathrm{T}, l, n}+\sum_{l \in \Pi_{u, n}}\left(\sum_{k \in S_{l, n}} \frac{G_{k, i}}{G_{k, l} G_{i, j}} C_{\mathrm{B}, l, n}\right)}{\beta_{d} N_{j, n} P_{\mathrm{T}, j, n}+\sum_{i \in S_{j, n} l \in \Pi-\{j\}} \sum_{i, l} P_{\mathrm{T}, l, n}+\sum_{i \in S_{j, n}} \sum_{l \in \Pi_{u, n}} \sum_{k \in S_{l, n}} \frac{G_{k, i}}{G_{i, j}} \frac{C_{\mathrm{B}, l, n}}{G_{k, l}}}
$$

TABLE II

MAXIMUM ACHIEVABLE $C I R$

\begin{tabular}{|c|c|c|c|c|}
\hline Load & 2 & 4 & 8 & 16 \\
\hline simplified link-gain matrix & -6.53 & -12.61 & -18.67 & -24.87 \\
\hline normal link-gain matrix & -6.53 & -12.61 & -18.67 & -24.87 \\
\hline
\end{tabular}

a cell load of 2 mobiles/cell/slot were used. This result may be exploit by channel allocation schemes to increase the system capacity by allowing crossed slots.

\section{Conclusions}

In this paper, a simplified generic optimum power control has been studied for uplink, downlink and crossed slots. The optimum power control is formulated into an eigenvalue problem using a simplified link-gain matrix. To simplify the link-gain matrix in downlink, the power control is applied to the total transmitted power and not to the communicationspecific power. Alternately, all communication links in uplink are received with the same power to verify the $C I R$ balancing in each cell. The simplification of the link-gain matrix decreases drastically the computation time and gives the same maximum achievable $C I R$. The proposed scheme can be used in FDD mode also. In this case, the gain in computation time will increase due the high allowed cell load in the FDD mode.

Moreover, a simplified link-gain matrix has been proposed for crossed slots. Simulation results has shown that crossed slots have usually the worst performance. However, the performance during the latter slots is better than the performance during uplink slots for some mobile distributions especially for low loads and low values of the asymmetry rate. Therefore, intelligent allocation technique must be used to efficiently exploit this type of distribution and to retain the flexibility of the TDD mode.

Due to the drastic reduction in the computation time and to the crossed slots integration, the proposed scheme can be used to develop sophisticated channel allocation technique. In TDD mode, the interference power in a server is broadcast to all the mobiles in the server. Therefore, an uplink decentralized power control based on the proposed optimal power control with the consideration of thermal noise is one of our future work.

\section{REFERENCES}

[1] A. J. Viterbi, CDMA: Principles of Spread Spectrum communication. Reading, MA: Addison-Wesley, 1995.

[2] J. Zander, "Performance of optimum transmitter power control in cellular radio systems," IEEE Transactions on Vehicular Technology, vol. 41, no. 1 , pp. $57-62,1992$.

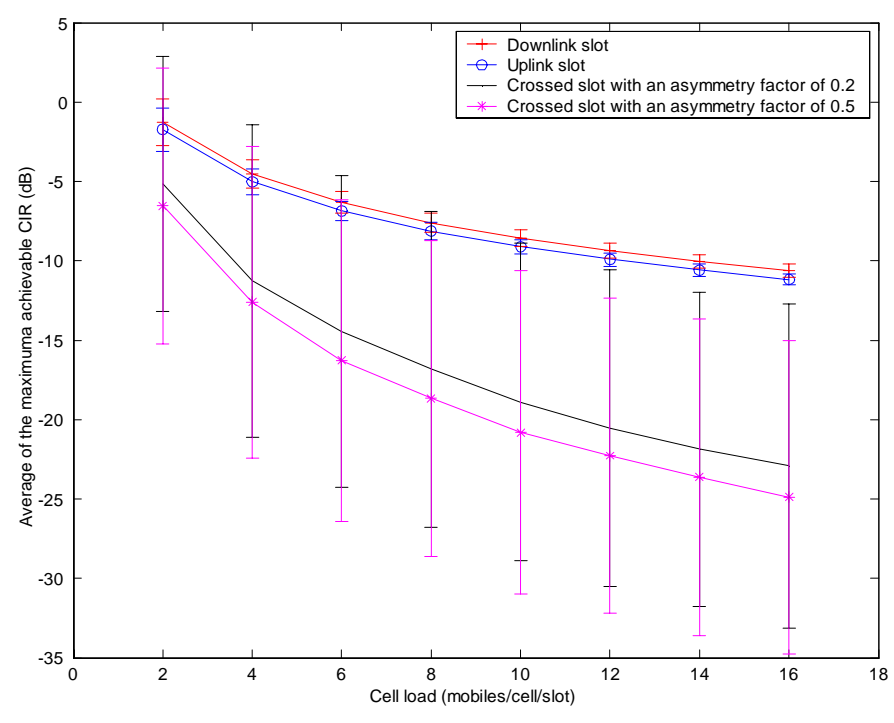

Fig. 2. The average maximum achievable $C I R$ (in $\mathrm{dB}$ ) as a function of the number of active mobiles in a cell during downlink, uplink and crossed slots.

[3] J. M. Aein, "Power balancing in systems employing frequency reuse," COMSAT tech. rev., vol. 3, no. 2, 1973.

[4] Q. Wu, "Performance of optimum transmitter power control in CDMA cellular mobile systems," IEEE Transactions on Vehicular Technology, vol. 48 , no. 2 , pp. $571-575,1999$.

[5] H. Haas and S. McLaughlin, "A dynamic channel assignment algorithm for a hybrid TDMA/CDMA-TDD interface using the novel TS-opposing technique," IEEE Journal on Selected Areas in Communications, vol. 19, no. 10 , pp. $1831-1846,2001$.

[6] J.-H. Wen, J.-S. Sheu, and J.-L. Chen, "An optimum downlink power control method for cdma cellular mobile systems," in Proc. of the IEEE International Conference on Communications, vol. 6, 2001, pp. 1738 1742 .

[7] J. Nasreddine and X. Lagrange, "Power control and slot allocation in a TD-CDMA system," in Proc. of the 55th IEEE Vehicular Technology Conference, 2002, pp. 880-884.

[8] J.-H. Wen, J.-S. Sheu, J.-Y. Wang, J.-L. Chen, and T.-K. Woo, "A feasible method to implement optimum CIR-balanced power control in CDMA cellular mobile systems," IEEE Transactions on Vehicular Technology, vol. 52 , no. 1 , pp. $80-95,2003$.

[9] J. Nasreddine and X. Lagrange, "Comparison of different slot allocation schemes in a TD-CDMA TDD system," in Proc. of the second IEEE Workshop on Applications and Services in Wireless Networks, 2002.

[10] S. Verdu, Multiuser detection. Cambridge University Press, 1998.

[11] F. R. Gantmacher, The theroy of matrices. New York: Chelsea publishing company, 1971, vol. 2.

[12] J. Zander and M. Frodigh, "Comment on 'performance of optimum transmitter power control in cellular radio systems'," IEEE Transactions on Vehicular Technology, vol. 43, no. 3, p. 636, 1994.

[13] S. AG, "TDD UE-UE interference simulations," TSG-RAN Working Group 4 (Radio meeting n26), 2003.

[14] J. Nasreddine and X. Lagrange, "Time slot allocation based on path gain division scheme for TD-CDMA TDD systems," in Proc. of the 57th IEEE Semiannual Vehicular Technology Conference, vol. 2, 2003, pp. $1410-$ 1414 . 